\title{
Associations between YKL-40 and markers of disease severity and death in patients with necrotizing soft-tissue infection
}

\author{
Morten Hedetoft ${ }^{*}{ }^{*} \mathbb{D}$, Marco Bo Hansen ${ }^{1,2}$, Martin Bruun Madsen ${ }^{3} \mathbb{C}^{0}$, Julia Sidenius Johansen ${ }^{4,5,6}$ and \\ Ole Hyldegaard ${ }^{1}$ D
}

\begin{abstract}
Background: Necrotizing soft-tissue infection (NSTI) is a severe and fast-progressing bacterial infection. Prognostic biomarkers may provide valuable information in treatment guidance and decision-making, but none have provided sufficient robustness to have a clinical impact. YKL-40 may reflect the ongoing pathological inflammatory processes more accurately than traditional biomarkers as it is secreted by the activated immune cells, but its prognostic yields in NSTI remains unknown. For this purpose, we investigated the association between plasma YKL-40 and 30-day mortality in patients with NSTI, and assessed its value as a marker of disease severity.
\end{abstract}

Methods: We determined plasma YKL-40 levels in patients with NSTI $(n=161)$ and age-sex matched controls $(n=65)$ upon admission and at day 1,2 and 3.

Results: Baseline plasma YKL-40 was $1191 \mathrm{ng} / \mathrm{mL}$ in patients with NSTI compared with $40 \mathrm{ng} / \mathrm{mL}$ in controls $(p<0.001)$. YKL-40 was found to be significantly higher in patients with septic shock (1942 vs. $720 \mathrm{ng} / \mathrm{mL}, p<0.001)$, and in patients receiving renal-replacement therapy $(2382 \mathrm{vs.} 1041 \mathrm{ng} / \mathrm{mL}, \mathrm{p}<0.001)$. YKL-40 correlated with Simplified Acute Physiology Score II (Rho 0.33, p<0.001). Baseline YKL-40 above $1840 \mathrm{ng} / \mathrm{mL}$ was associated with increased risk of 30-day mortality in age-sex-comorbidity adjusted analysis (OR 3.77, 95\% Cl; 1.59-9.24, $\mathrm{p}=0.003$ ), but after further adjustment for Simplified Acute Physiology Score II no association was found between YKL-40 and early mortality.

Conclusion: High plasma YKL-40 to be associated with disease severity, renal-replacement therapy and risk of death in patients with NSTI. However, YKL-40 is not an independent predictor of 30-day mortality.

Keywords: Chitinase-3-like-1 protein, Clinical endpoint, Fournier's gangrene, Necrotizing fasciitis, Sepsis, Survival

\section{Background}

Necrotizing soft-tissue infection (NSTI) is a rare, severe and fast-progressing bacterial infection [1]. The incidence rate of NSTI is approximately two per 100,000 inhabitants/year in Denmark [2], but varies markedly across countries [3-6]. NSTI can be caused by a myriad

\footnotetext{
*Correspondence: morten.friis.fiskbaek.hedetoft@regionh.dk

${ }^{1}$ Department of Anaesthesia, Centre of Head and Orthopaedics,

Copenhagen University Hospital, Rigshospitalet, Blegdamsvej 9,

2100 Copenhagen, Denmark

Full list of author information is available at the end of the article
}

of aerobic, anaerobic and facultative anaerobic bacteria [7], but predominantly by Group A Streptococcus in monomicrobial infections [8]. The clinical condition can rapidly progress into septic shock, multiple organ failure and death. Early recognition is key in NSTI, however timely diagnosis may be rendered as the initial symptoms such as swelling, pain and erythema are difficult to discriminate from less severe skin infections, consequently an initially misdiagnosis of $71 \%$ has been reported [9]. Despite early, radical and intensive multidisciplinary care 
the mortality remains high and largely unaltered in several years $[2,4]$.

Biomarkers may provide valuable information to the treating physician in treatment guidance and decisionmaking in NSTI patients, potentially improving morbidity and mortality. However, biomarkers have only been limited investigated in NSTI. No reliable diagnostic biomarkers exist $[10,11]$, hence surgical exploration persists the diagnostic golden standard although some imaging modalities may provide valuable diagnostic information [12-14]. Prognostic biomarkers have been sparsely assessed in patients with NSTI and none have provided sufficient robustness to have clinical impact [15-20]. Therefore, standard biochemistry and disease severity scores, such as Simplified Acute Physiology Score II (SAPS II), continues to provide the best level of prognostic risk-stratification and disease monitoring in the intensive care unit. Consequently, novel biomarkers are warranted in effort of increasing early diagnostic, therapeutic and prognostic risk-stratification in patients with NSTI.

YKL-40, also called chitinase-3-like-1 protein, may be an attractive prognostic biomarker in NSTI. YKL-40 is an acute phase protein secreted by various of immune cells, including macrophages, neutrophils and endothelial cells [21]. Of notice, proteomic analysis has indicated YKL-40 as a promising biomarker in patients with severe sepsis and septic shock [22]. Studies have suggested that high YKL-40 levels could be prognostic in patients with sepsis $[23,24]$. However, the prognostic yields of YKL40 remain unknown in NSTI. Extrapolation of YKL-40 results from patients with sepsis to NSTI may be questionable as the inflammatory response differ due to the combination of extensive tissue damage in NSTI and different pathogenesis in general. It would be of interests if high plasma YKL-40 levels could identify patients with NSTI with an increased risk of death as these patients might benefit from a more aggressive treatment protocol.

Therefore, we aimed to assess plasma YKL-40 as a prognostic biomarker in patients with NSTI and evaluate its accuracy as marker of disease severity and mortality. We hypothesized that high plasma levels of YKL-40 are associated with disease severity and 30-day mortality.

\section{Methods}

\section{Study design and population}

The present study was based on data of the Danish fraction from the prospective, observational INFECT study [8] (ClinicalTrials.gov; NCT01790698). We included patients with NSTI referred to Copenhagen University Hospital (Rigshospitalet, a tertiary hospital with a national NSTI service treatment), from February 2013 to October 2015.
We included adult patients ( $\geq 18$ years) with a surgical confirmed NSTI diagnosis at either primary operation or at revision. We excluded patients in whom surgery did not reveal signs of NSTI. Control patients consisted of age-sex matched elective orthopedic patients without ongoing infection or inflammatory conditions (included between September 2014 and March 2015) [25]. The Strengthening the Reporting of Observational Studies in Epidemiology (STROBE) guidelines were followed in drafting of the present manuscript [26].

\section{Patient management}

Our standardized multidisciplinary treatment protocol has in details been reported elsewhere [8]. In brief, the patients received frequent surgical debridement, initial broad-spectrum antibiotics (Clindamycin, Ciprofloxacin and Meropenem), intensive supportive care, hyperbaric oxygen treatment and immunoglobin G.

\section{Data collection}

Predefined data were registered by dedicated personnel into an electronic database [27]. For the present study, clinical data included age, sex, comorbidities (cardiovascular disease, chronic kidney disease, chronic obstructive pulmonary disease, diabetes, immune deficiency, chronic liver disease, malignancy, peripheral vascular disease and rheumatoid disease), pre-existing condition (smoking, alcohol consumption, steroid treatment and immunosuppressing drugs), microorganism, standard biochemistry, severity scores (Simplified Acute Physiology Score II [SAPS II] and Sequential Organ Failure Assessment [SOFA]), ICU-length of stay, septic shock, amputation, renal-replacement therapy and vital status at day 30 .

\section{YKL-40 measurement}

Patient had blood samples collected into ethylenediaminetetraacetic acid (EDTA) sample tubes upon admission (baseline), and at the following three days (all between 8 a.m. and 12 a.m.). Samples were immediately put on ice and within $40 \mathrm{~min}$ centrifugated at $3500 \mathrm{rpm}$ (2400G) for $10 \mathrm{~min}$. The separated plasma was collected into cryotubes and stored at $-80^{\circ} \mathrm{C}$ until analysis.

Plasma YKL-40 was quantified in duplicates by commercial enzyme-linked immunosorbent (ELISA) technique (Quidel, San Diego, CA, USA) at each of the four time points. The minimal detectable limit for YKL-40 was $10 \mathrm{ng} / \mathrm{ml}$. The intra-assay CV was $<5 \%$ and the interassay CV was $<6 \%$ [28]. Plasma YKL-40 concentration was determined at Herlev Hospital without knowledge of the clinical data and prognosis of the patients. 


\section{Outcomes}

The primary outcome was association between baseline YKL-40 and 30-day mortality. Secondary analyses included the association between baseline YKL-40 and septic shock, renal-replacement therapy (RRT), amputation, SAPS II, SOFA score at day 1 and baseline blood lactate. We planned to run a sub-group analysis on YKL-40 levels according to infection with or without Group A Streptococcus.

\section{Statistical analysis}

Test for normally were assessed with Shapiro-Wilks Test. Due to nonparametric distribution; continuous data are presented as medians (interquartile range, IQR) and categorial data as absolute numbers (percentage \%). Continuous data were compared using Wilcoxon Rank Sum Test whereas categorical data were compared using Fisher's Exact Test. We assessed correlations by Spearman's rank correlation test. Receiver operating characteristic (ROC) curves were analyzed for baseline YKL-40 levels on 30-day mortality and area-under-curve (AUC) were reported. We categorized low versus high plasma YKL-40 level at admission according to both the median and the Youden Index optimal cutoff point [29]. Sensitivity, specificity, positive-predictive value (PPV) and negative-predictive value (NPV) were reported as prognostic test accuracy. Logistic regression analyses with Odds Ratio (OR) and 95\% confidence intervals (95\% CI) were used to assess the association between baseline YKL-40 and 30-day mortality. We adjusted for differences in age, sex, comorbidities (yes/no) and SAPS II score. Patients with missing SAPS II scores were excluded from logistic regression analyses. P-values are reported as exact unless $<0.001$. P-values $<0.05$ was considered statistically significant. Statistical analyses performed using RStudio Version 1.0.153 (RStudio, Inc.). Figures prepared in GraphPad Prism Version 8.0.2. (GraphPad Inc., La Jolla, CA, USA).

\section{Results}

A total of 161 patients with NSTI (Additional file 1) and 65 age- and sex matched controls were included in the study. The clinical characteristics of the patients are presented in Table 1. No patients were lost to follow-up at day 30. Very high plasma YKL-40 levels were found at admission and at day 1, 2 and 3: $1191 \mathrm{ng} / \mathrm{mL}$ (IQR 538-2387), $1418 \mathrm{ng} / \mathrm{mL}$ (IQR 651-2445), $816 \mathrm{ng} /$ $\mathrm{mL}$ (IQR 436-1798) and $537 \mathrm{ng} / \mathrm{mL}$ (IQR 261-1053), respectively. The controls had a baseline plasma YKL40 of $40 \mathrm{ng} / \mathrm{mL}$ (IQR 29-87). Plasma YKL-40 was significantly higher in patients with NSTI compared to
Table 1 Characteristics of patients with necrotizing soft-tissue infection

Entire cohort $(n=161)$

\begin{tabular}{|c|c|}
\hline \multicolumn{2}{|l|}{ Demographics } \\
\hline Age, years & $61(53-69)$ \\
\hline Sex, male & $98(61)$ \\
\hline Body mass index, $\mathrm{kg} / \mathrm{m}^{2}$ & $26(24-31)$ \\
\hline \multicolumn{2}{|l|}{ Comorbidities } \\
\hline Cardiovascular disease & $75(47)$ \\
\hline Chronic kidney disease & $13(8)$ \\
\hline COPD & $17(11)$ \\
\hline Diabetes & $40(25)$ \\
\hline Immune deficiency & $12(7)$ \\
\hline Chronic liver disease & $9(6)$ \\
\hline Malignancy & $14(9)$ \\
\hline Peripheral vascular disease & $21(13)$ \\
\hline Rheumatoid disease & $11(7)$ \\
\hline No comorbidities & $49(30)$ \\
\hline \multicolumn{2}{|l|}{ Pre-existing conditions } \\
\hline Active smoker ${ }^{a}$ & $49(30)$ \\
\hline High alcohol consumption ${ }^{b}$ & $23(14)$ \\
\hline Steroid treatment & $21(13)$ \\
\hline Immunosuppressing drugs & $15(9)$ \\
\hline \multicolumn{2}{|l|}{ Biochemistry } \\
\hline Leukocyte count, $10^{9} / \mathrm{L}$ & $16.2(11.4-22.7)$ \\
\hline C-reactive protein, mg/L & $224(153-309)$ \\
\hline Procalcitonin, $\mu \mathrm{g} / \mathrm{L}$ & $8.6(2.1-37.1)$ \\
\hline Creatinine, $\mu \mathrm{mol} / \mathrm{L}$ & $121(79-307)$ \\
\hline Lactate, $\mathrm{mmol} / \mathrm{L}$ & $2.3(1.3-4.7)$ \\
\hline \multicolumn{2}{|l|}{ Other } \\
\hline SOFA score ${ }^{c}$ & $8(6-11)$ \\
\hline SAPS $\|^{d}$ & $45(35-54)$ \\
\hline Septic shock ${ }^{e}$ & $80(50)$ \\
\hline ICU length of stay, days ${ }^{f}$ & $7(4-13)$ \\
\hline Amputation within 7 days $^{9}$ & $27(17)$ \\
\hline RRT within 7 days & $35(22)$ \\
\hline 30-day mortality, n (\%, 95\% Cl) & $28(17,12-24)$ \\
\hline 90-day mortality, n (\%, 95\% Cl) & $37(23,17-30)$ \\
\hline
\end{tabular}

Continuous data are presented as medians (interquartile range, IQR) and categorial data as absolute numbers (percentage, \%)

$C O P D$ chronic obstructive pulmonary disease, $I C U$ intensive care unit, $R R T$ renal replacement therapy

a Data were missing for 38 (24\%) patients. ${ }^{\text {b } H i g h ~ a l c o h o l ~ c o n s u m p t i o n ~ d e f i n e d ~}$ as $>14$ units of alcohol/week (female); $>21$ units of alcohol/week (male), data were missing for 43 (27\%) patients. 'Sequential Organ Failure Assessment (SOFA) Score (Day 1); data were missing for $3(2 \%)$ patients. ${ }^{d}$ Simplified Acute Physiology Score II (SAPS II); data were missing for 4 (2\%) patients. ${ }^{\text {EFrom }}$ the first $24 \mathrm{~h}$ after admission. Septic shock defined as lactate $>2 \mathrm{mmol} / \mathrm{l}$ and

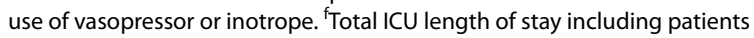
subsequently transferred to ICUs in other hospitals. ${ }^{9} 1$ (50\%) patients had NSTI on an extremity 

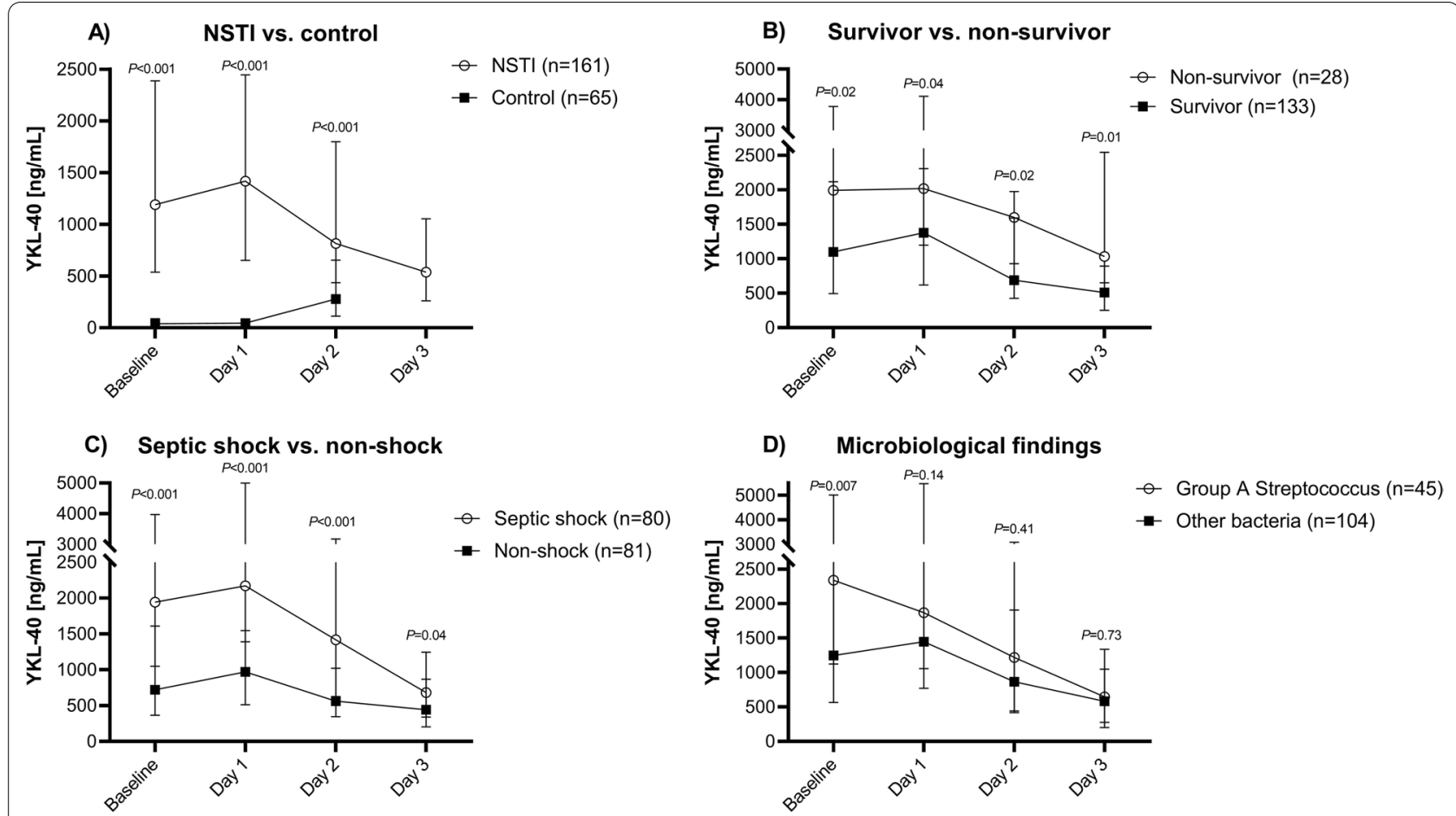

Fig. 1 Plasma YKL-40 levels at admission (baseline), day 1, day 2 and day 3 in a NSTI vs. age- and sex-matched controls, b 30-day survivors vs. non-survivors, c septic shock vs. non-shock and $\mathbf{d}$ presence of Group A Streptococcus in blood and/or tissue. Note the two-segmented y-axis. Data are presented as medians with interquartile range. Comparisons performed by Wilcoxon Rank Sum Test. Septic shock was defined as s-lactate $>2$ and use of vasopressor/inotropic agents. Microbiological findings were classified according to our protocol, in short, presumed pathogenic agent isolated at primary operation or immediately prior, up to three days after diagnosis. Group A streptococcus includes both monomicrobial GAS infections and polymicrobial infections including GAS. N=12 patients had no positive microbiological findings

controls at admission, and at day 1 and 2 (Fig. 1A; controls had no YKL-40 determination at day 3).

\section{Association between YKL-40 and mortality}

A total of $28(17 \%)$ patients died within 30 days after admission. Among non-survivors, the median SAPS II score was 63 (51-77), and 18 patients had septic shock upon admission as compared to $41(33-51)$ and 62, respectively, in survivors. In non-survivors, plasma YKL40 at admission was significantly higher compared to survivors (1992 ng/mL (IQR 1107-3780) versus $1100 \mathrm{ng} / \mathrm{mL}$ (IQR 496-2117), $\mathrm{p}=0.02$ ). This finding persisted at day 1 , 2 and 3 (Fig. 1B).

The optimal- and median cut-off levels of plasma YKL40 at admission were $1840 \mathrm{ng} / \mathrm{mL}$ and $1191 \mathrm{ng} / \mathrm{mL}$, respectively. In univariate analyses, plasma YKL-40 levels above the median and the optimal cut-off point were statistically associated with increased mortality (Table 2). These findings persisted in age, sex and comorbidity adjusted analyses, however when additionally adjusted for SAPS II no significant associations were observed (Table 2).
Plasma YKL-40 at admission showed a ROC-AUC of 0.64 (95\% CI 0.52-0.76) on 30-day mortality (Additional file 2). This was lower than the SAPS II ROC-AUC of 0.86 (95\% CI $0.80-0.93)$ and plasma lactate of 0.80 (95\% CI $0.71-0.89)$. The prognostic accuracy of high YKL-40 is presented in Table 3.

\section{Association between YKL-40 and severity of disease}

Plasma YKL-40 increased in most patients from admission to day $1, \mathrm{p}<0.001$ (a median of $14 \mathrm{~h}$ (IQR 9-19) were observed from blood sampling at admission to day 1). Thereafter, plasma YKL-40 decreased from day 1 to day 2 and 3 (Fig. 1A). Admission plasma YKL-40 correlated with SAPS II and SOFA score, and with plasma lactate and creatinine (Table 3 ).

Plasma YKL-40 at admission was significantly higher in patients with septic shock $(\mathrm{n}=80)(1942 \mathrm{ng} / \mathrm{mL}$ (IQR 1048-3970) versus $720 \mathrm{ng} / \mathrm{mL}$ (IQR 366-1610), $\mathrm{p}<0.001)$ in non-shock patients $(\mathrm{n}=81)$ (Fig. 1C). This finding persisted on day 1, 2 and 3 (Fig. 1C). Moreover, patients receiving RRT within 7 days had significantly higher YKL-40 compared to patient not requiring RRT (Fig. 2). Plasma YKL-40 at admission was significantly 
Table 2 Univariate and multivariate logistic regression analyses of 30-day mortality based on plasma YKL-40 concentration at admission according to the median and optimal cut-off values

\begin{tabular}{|c|c|c|c|c|c|c|c|c|c|c|}
\hline & \multirow[t]{2}{*}{$\begin{array}{l}\text { Plasma YKL-40 } \\
\mathrm{ng} / \mathrm{mL}\end{array}$} & \multicolumn{3}{|c|}{ Unadjusted } & \multicolumn{3}{|c|}{$\begin{array}{l}\text { Adjusted analysis: age, sex and } \\
\text { comorbidity }^{\mathrm{a}}\end{array}$} & \multicolumn{3}{|c|}{ 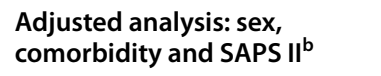 } \\
\hline & & OR & $95 \% \mathrm{Cl}$ & $P$ & OR & $95 \% \mathrm{Cl}$ & $P$ & OR & $95 \% \mathrm{Cl}$ & $P$ \\
\hline \multirow[t]{2}{*}{ Median } & $<1191$ & & 1 (Reference) & & & 1 (Reference) & & & 1 (Reference) & \\
\hline & $\geq 1191$ & 3.00 & $1.27-7.69$ & 0.02 & 3.13 & $1.30-8.19$ & 0.01 & 1.45 & $0.51-4.24$ & 0.49 \\
\hline \multirow[t]{2}{*}{ Optimal } & $<1840$ & & 1 (Reference) & & & 1 (Reference) & & & 1 (Reference) & \\
\hline & $\geq 1840$ & 3.55 & $1.55-8.48$ & 0.003 & 3.77 & $1.59-9.24$ & 0.003 & 1.75 & $0.62-4.89$ & 0.29 \\
\hline
\end{tabular}

CI confidence interval, OR odds ratio, SAPS II simplified acute physiology score II (data were missing for 4 (2\%) patients, all survivors, these patients were excluded from the analyses)

${ }^{a}$ Comorbidity dichotomized (yes/no)

${ }^{\mathrm{b}}$ Age included in SAPS II score

Table 3 Left: Diagnostic accuracy of YKL40 (defined by Youden's optimal cut-off point) in predicting 30-day mortality. Right: Spearman Rank Correlation between disease severity scores and baseline YKL-40

\begin{tabular}{|c|c|c|c|c|}
\hline \multicolumn{2}{|l|}{ Accuracy } & \multicolumn{3}{|l|}{ Correlation } \\
\hline & & & Rho & $P$ \\
\hline Sensitivity & $0.61(95 \% \mathrm{Cl} 0.41-0.78)$ & & & \\
\hline Specificity & $0.70(95 \%$ Cl $0.61-0.77)$ & SAPS $\|$ & 0.33 & $<0.001$ \\
\hline PPV & $0.30(95 \%$ Cl $0.18-0.43)$ & SOFA Score & 0.48 & $<0.001$ \\
\hline NPV & 0.89 (95\% Cl 0.82-0.95) & Lactate & 0.49 & $<0.001$ \\
\hline AUC-ROC & 0.64 (95\% Cl 0.52-0.76) & Creatinine & 0.46 & $<0.001$ \\
\hline
\end{tabular}

Data are presented as fractions (95\% Confidence Interval)

$A U C-R O C$ area under the receiver operating characteristics curve, $\mathrm{Cl}$ confidence interval, NPV negative predictive value, $P P V$ positive predictive value, SAPS II simplified acute physiology score II, SOFA Score sequential organ failure assessment score day 1

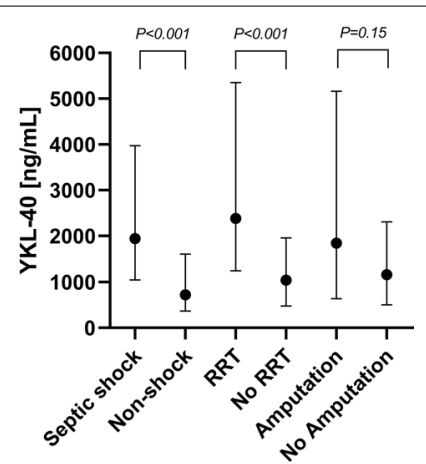

Fig. 2 Plasma YKL-40 concentrations at admission in patients with necrotizing soft tissue infection according to patients with septic shock versus non-shock; RRT versus no RRT; and amputation versus non amputation (81 patients had NSTI located on an extremity). Data are presented as medians with interquartile range. Comparisons performed with Wilcoxon Rank Rum Test. RRT renal-replacement treatment higher among patients infected with mono or polymicrobial Group A Streptococcus $(\mathrm{n}=45)$ in blood and/or tissue (2338 ng/mL, IQR 1123-5007) compared to other types of NSTI $(\mathrm{n}=104)(1246 \mathrm{ng} / \mathrm{mL}, \mathrm{IQR} 565-2749)$, $\mathrm{p}=0.007$. However, this was not observed at day 1,2 and 3 (Fig. 1D). No microbial findings were observed in 12 patients.

\section{Discussion}

In this study, assessing the prognostic yields of plasma YKL-40 in patients with NSTI, we observed high plasma YKL-40 levels to be associated with increased risk of death and severity of the disease. However, the association was not statistically significant when adjusted for SAPS II. We found the highest plasma YKL-40 levels at admission among patients in septic shock, patients requiring RRT and patients infected with Group A Streptococcus.

We compared the prognostic accuracy of 30-day mortality of plasma YKL-40 with plasma lactate, known to be associated with increased mortality $[8,15]$, and with SAPS II which is among the most common disease severity score used in the intensive care unit. We found a lower ROC-AUC for YKL-40 compared to both plasma lactate and SAPS II, indicating that the prognostic information of plasma YKL-40 is not be better than the existing evaluation tools. Yet, caution is required when exclusively comparing AUC between prognostic indicators, as AUC reflects the overall performance of all possible cut-off values including those that will never be clinically applied. Therefore, we addressed the prognostic accuracy as sensitivity, specificity, PPV and NPV according to the optimal cut-off point. Here we observed a low sensitivity but moderate good specificity and NPV indicating patients with plasma YKL-40 levels below $1840 \mathrm{ng} / \mathrm{mL}$ to have an $89 \%$ chance of surviving the first 30 days. We observed a reduction in YKL-40 over time potentially reflecting a 
generally improved clinical condition among patients, but such result must be cautiously interpreted as a risk of dilution occurs when measuring across time, as the most critically ill patients who have highest YKL-40 levels at admission, are probably also the patients who first decease.

To our knowledge, no prognostic biomarkers have yet provided enough robustness to provide a clinical impact in NSTI treatment. Pentraxin-3, a molecule released by various immune cells at the onset of inflammation, has earlier demonstrated a ROC-AUC of 0.66 in predicting 180-day mortality with a sensitivity of 0.69 and a specificity of 0.56 in patients with NSTI [18]. Additionally, the prognostic accuracy of suPAR in predicting 90-day mortality has shown a ROC-AUC of 0.77 , but the combination of suPAR and SAPS II was not superior compared to SAPS II alone (ROC-AUC 0.87) [17]. The burden of disease, the unaltered mortality rates and the lack of robust biomarkers all highlight the need for studies evaluating novel biomarkers potentially capable of reducing disease morbidity and mortality.

We included age, sex, comorbidity and disease severity as covariates in logistic regression analyses, as these are among the most documented prognostic risk factors of not surviving NSTI [2, 4, 30-36]. Other covariates such as septic shock $[34,37]$ need of inotropes and RRT [35, 38] could have been included as covariates, but according to the 'one-in-ten rule' on the number of covariates in logistic analyses, only three covariates entered multivariate analyses $[39,40]$. Furthermore, these are included in the SAPS II. We observed high plasma YKL-40 at admission to be associated with increased mortality in univariate and age-sex adjusted analyses, but not after adjusting for SAPS II indicating that YKL-40 is not an independent predictor of mortality. Yet, plasma YKL-40 could provide an early prognostic information for the treating physician as SAPS II needs $24 \mathrm{~h}$ of admission in the intensive care unit before a complete score can be calculated, and some variables may be missing rendering the calculation. Although no quick/bed-side YKL-40 assays exist, wellperforming and rapid multiplex assays $(\approx 75 \mathrm{~min})$ have been developed [41].

Severe infection such as patients with septic shock and patients requiring RRT are associated with mortality $[34,37,38]$. Therefore, we compared differences in plasma YKL-40 according to presence of septic shock or requiring RRT. We found patients with septic shock to have higher plasma YKL-40 levels at admission and the following three days compared to the levels in non-shock patients. Similar patterns were observed in patients requiring RRT and patients not receiving RRT. These results may reflect a more pronounced immune cell activity and a higher burden of disease in these patients.
Additionally, patients with Group A Streptococcus were found to have significantly higher plasma YKL-40 levels at admission compared to patients with other types of NSTI, potentially reflecting a higher rate of septic shock in these individuals as earlier demonstrated [8]. These observations suggest that burden of disease is increased in patients with high plasma YKL-40 and is underlined by the positive correlations between baseline YKL-40 and SAPS II, SOFA score and plasma lactate in the present study.

In healthy individuals, plasma YKL-40 is approximately $40 \mathrm{ng} / \mathrm{mL}(2.5-97.5 \%$ CI 14-155) [28] which is comparable to our observation of $40 \mathrm{ng} / \mathrm{mL}$ (IQR 29-87). We demonstrated approximately 30 times higher plasma levels in patients with NSTI compared to controls, indicating that severe bacterial infections influence YKL40 secretion from inflammatory cells. Others have earlier found plasma YKL-40 levels in patients with sepsis and septic shock to be approximately 700-1000 and 1200-2200 ng/mL, respectively [23]. These results are in accordance with the present plasma YKL-40 levels of $720 \mathrm{ng} / \mathrm{mL}$ in non-shock NSTI patients and $1942 \mathrm{ng} /$ $\mathrm{mL}$ in NSTI patients with septic shock. Additionally, in a cohort of 89 patients with Streptococcus pneumoniae bacteremia the median YKL-40 was $342 \mathrm{ng} / \mathrm{mL}$ and was associated with in-hospital mortality [42], whereas in 289 hospitalized patients with community-acquired pneumonia, plasma YKL-40 was higher in patients with severe infection and associated with short- and longterm mortality [43]. Interestingly, a study including both experimental and human data has revealed that YKL-40 may be able to discriminate been sepsis-induced acute kidney injury and sepsis without acute kidney injury [44]. In that context, patients with Puumala hantavirus infection-a virus infection typically inducing acute kidney injury-YKL-40 was more pronounced during the acute phase, and correlated with the degree of inflammation and severity of acute kidney injury [45]. These finding are in agreement with the present study demonstrating significantly higher levels plasma YKL-40 in those receiving RRT within 7 days from admission.

Furthermore, plasma YKL-40 has recently been demonstrated to be a biomarker of future infectious diseases in the general population with adjusted hazard ratio of future episodes of skin infections and sepsis of 1.76 and 1.90 , respectively [46]. These findings emphasize plasma YKL-40 as a promising biomarker for further research in infectious diseases including NSTI.

YKL-40 is not a sole biomarker for infectious disease [21]. Plasma YKL-40 has been studied in patients with different types of cancer and high levels are associated with short overall survival [47]. Furthermore, high plasma YKL-40 levels are also associated with poor 
prognosis in non-cancerous diseases including cardiovascular, respiratory, digestive, endocrine, nervous, urinary and skeletal diseases [21]. In patients with infectious disease plasma YKL-40 may reflect the ongoing pathological inflammatory processes more accurately than traditionally biomarkers such as CRP (produced by hepatocytes), since YKL-40 is secreted by activated immune cells including macrophages, neutrophils and endothelial cells [21]. YKL-40 is considered an acute-phase protein and is stimulated by INF- $\gamma$ and IL-6 $[48,49]$. We have earlier demonstrated plasma IL- 6 to be associated with NSTI disease severity and particular elevated in patients with septic shock and $\beta$-hemolytic streptococcal infection, potentially indicating a relationship between YKL-40/ IL6 and burden of disease in NSTI [20]. Despite some regulators of YKL-40 have been defined during the last decades [21], a great knowledge gap exists on its pathophysiological role and functional effects in infectious diseases including sepsis and NSTI [50, 51]. Future research may include evaluation of YKL-40 in non-necrotic softtissue lesions such as erysipelas, cellulitis or cutaneous abscesses in effort of investigating the diagnostic yields of YKL-40.

To our knowledge this is the first study to report plasma YKL-40 levels in patients with NSTI. A strength of the present study is the sample size and that no patients were lost to follow-up. We had broad inclusion criteria and only few exclusion criteria. Additionally, the clinical data was prospectively collected by dedicated personnel using a predefined and standardized protocol [27]. Lastly, the laboratory conducting the plasma YKL-40 analyses was blinded for patient type and outcome. However, some limitations exist: First, since this is an observational study there is a potential risk of missing unknown confounders. Second, although our study center has a national NSTI service treatment and thereby increasing the heterogeneity, we may be missing the patients who are too ill for transportation consequently introducing an important selection bias into our analyses. Third, several patients were either on immunosuppressing drugs or steroid treatment at admission theoretically impacting YKL-40 concentration. Last, no predefined protocol regarding biomarker analyses was published.

\section{Conclusions}

We found that plasma YKL-40 levels were associated with disease severity and risk of death in patients with NSTI. However, YKL-40 is not an independent predictor for 30-day mortality.

\section{Abbreviations}

AUC: Area-under-curve; Cl: Confidence interval; ELISA: Enzyme-linked immunosorbent assay; IQR: Interquartile range; NSTI: Necrotizing soft-tissue infection; NPV: Negative-predictive value; OR: Odds ratio; PPV: Positive-predictive value; ROC: Receiver operating characteristic; RRT: Renal-replacement therapy; SAPS II: Simplified acute physiology score II; SOFA: Sequential organ failure assessment.

\section{Supplementary Information}

The online version contains supplementary material available at https://doi. org/10.1186/s12879-021-06760-x.

Additional file 1. Flow chart of patients included in the study. Patients with suspected NSTI were screened for eligibility. Patients were excluded if they did not meet the criteria of inclusion. After inclusion, patients'files were reviewed and 7 were deemed non-NSTI due to no intraoperative signs of necrotizing soft tissue infection. 2 patients did not have blood samples available for analyses. 1 patient was discontinued as informed consent was not obtainable.

Additional file 2. Receiver operating characteristics curve of 30-day mortality in patients with necrotizing soft-tissue infection according to plasma YKL-40 and lactate at admission and SAPS II. SAPS II; Simplified Acute Physiology Score II.

\section{Acknowledgements}

None.

\section{Authors' contributions}

Study planning $(\mathrm{MH}, \mathrm{MBH}, \mathrm{JS}, \mathrm{OH})$, data analysis $(\mathrm{MH}, \mathrm{OH})$, results interpretation $(\mathrm{MH}, \mathrm{MBH}, \mathrm{MBM}, \mathrm{JS} J, \mathrm{OH})$, drafting manuscript $(\mathrm{MH}, \mathrm{OH})$. The corresponding author attests that all listed authors meet authorship criteria and that no others meeting the criteria have been omitted. Corresponding author serves as guarantor for the present study. All authors read and approved the final manuscript.

\section{Funding}

The study was supported by the projects of PERMIT (Grant Number 811300009B) funded by Innovation Fund Denmark and EU Horizon 2020 under the frame of ERA PerMed (project 2018-151) and PERAID (Grant Number 8114-00005B) funded by Innovation Fund Denmark and Nordforsk (project no. 90456). The research leading to these results has received funding from the European Union Seventh Framework Programme: (FP7/2007-2013) under the grant agreement 305340 (INFECT project). Moreover, Copenhagen University Hospital (Rigshospitalet) provided a research grant for MH (Grant Number R167-A7352-B3897). No funders had any role in the design, conduct, collection, analysis, and interpretation of the data. Nor in the preparation, approval or submission of the manuscript.

\section{Availability of data and materials}

The datasets analysed during the current study available from the corresponding author on reasonable request.

\section{Declarations}

\section{Ethics approval and consent to participate}

The study was approved by the Regional Ethical Committee of Capital Region $(\mathrm{H}-2-2014-071)$ and the Danish Data Protection Agency (no. 30-1282). Written informed consent was obtained from all patients or their legal surrogates. This study and all methods have been performed in accordance with the Declaration of Helsinki.

\section{Consent for publication}

Not applicable.

Competing interests

The authors declared that they do not have any competing interests.

Author details

${ }^{1}$ Department of Anaesthesia, Centre of Head and Orthopaedics, Copenhagen University Hospital, Rigshospitalet, Blegdamsvej 9, 2100 Copenhagen, 
Denmark. ${ }^{2}$ Konduto ApS, Sani nudge, Copenhagen, Denmark. ${ }^{3}$ Department of Intensive Care 4131, Copenhagen University Hospital, Rigshospitalet, Copenhagen, Denmark. ${ }^{4}$ Department of Medicine, Herlev and Gentofte Hospital, Copenhagen University Hospital, Copenhagen, Denmark. ${ }^{5}$ Department of Oncology, Herlev and Gentofte Hospital, Copenhagen University Hospital, Copenhagen, Denmark. ${ }^{6}$ Department of Clinical Medicine, Faculty of Health and Medical Sciences, University of Copenhagen, Copenhagen, Denmark.

\section{Received: 15 April 2021 Accepted: 1 October 2021} Published online: 09 October 2021

\section{References}

1. Stevens DL, Bryant AE. Necrotizing soft-tissue infections. N Engl J Med. 2017;377:2253-65.

2. Hedetoft M, Madsen M, Madsen L, Hyldegaard O. Incidence, comorbidity and mortality in patients with necrotising soft-tissue infections, 2005-2018: a Danish nationwide register-based cohort study. BMJ Open. 2020;10: e041302.

3. Naseer U, Steinbakk M, Blystad H, Caugant DA. Epidemiology of invasive group A streptococcal infections in Norway 2010-2014: a retrospective cohort study. Eur J Clin Microbiol Infect Dis. 2016;35:1639-48.

4. Audureau E, Hua C, de Prost N, Hemery F, Decousser JW, Bosc R, et al. Mortality of necrotizing fasciitis: relative influence of individual and hospital-level factors, a nationwide multilevel study, France, 2007-12. Br J Dermatol. 2017;177:1575-82.

5. Soltani AM, Best MJ, Francis CS, Allan BJ, Askari M, Panthaki ZJ. Trends in the incidence and treatment of necrotizing soft tissue infections: an analysis of the national hospital discharge survey. J Burn Care Res. 2014:35:449-54.

6. Khamnuan P, Chongruksut W, Jearwattanakanok K, Patumanond J, Tantraworasin A. Necrotizing fasciitis: epidemiology and clinical predictors for amputation. Int J Gen Med. 2015;8:195-202.

7. Peetermans M, de Prost N, Eckmann C, Norrby-Teglund A, Skrede S, De Waele JJ. Necrotizing skin and soft-tissue infections in the intensive care unit. Clin Microbiol Infect. 2020;26:8-17. https://doi.org/10.1016/j.cmi. 2019.06.031.

8. Madsen MB, Skrede S, Perner A, Arnell P, Nekludov M, Bruun T, et al. Patient's characteristics and outcomes in necrotising soft-tissue infections: results from a Scandinavian, multicentre, prospective cohort study. Intensive Care Med. 2019;45:1241-51.

9. Goh T, Goh LG, Ang CH, Wong CH. Early diagnosis of necrotizing fasciitis. Br J Surg. 2014;101:e119-25.

10. Urbina T, Madsen MB, de Prost N. Understanding necrotizing soft tissue infections in the intensive care unit. Intensive Care Med. 2020. https://doi. org/10.1007/s00134-020-06071-w.

11. Saccenti E, Svensson M. Systems biology and biomarkers in necrotizing soft tissue infections. In: Necrotizing soft tissue infections. Cham: Springer; 2020. p. 167-86.

12. Fernando SM, Tran A, Cheng W, Rochwerg B, Kyeremanteng K, Seely AJE, et al. Necrotizing soft tissue infection: diagnostic accuracy of physical examination, imaging, and LRINEC score: a systematic review and metaanalysis. Ann Surg. 2019;269:58-65.

13. Levenson RB, Singh AK, Novelline RA. Fournier gangrene: role of imaging. Radiographics. 2008;28:519-28.

14. Nougue H, Le Maho A-L, Boudiaf M, Blancal J-P, Gayat E, Le Dorze M, et al. Clinical and imaging factors associated with severe complications of cervical necrotizing fasciitis. Intensive Care Med. 2015;41:1256-63.

15. Schwartz S, Kightlinger E, de Virgilio C, de Virgilio M, Kaji A, Neville A, et al. Predictors of mortality and limb loss in necrotizing soft tissue infections. Am Surg. 2013;79:1102-5.

16. Yaghoubian A, de Virgilio C, Dauphine C, Lewis RJ, Lin M. Use of admission serum lactate and sodium levels to predict mortality in necrotizing soft-tissue infections. Arch Surg. 2007;142:840-6.

17. Polzik P, Grøndal O, Tavenier J, Madsen MB, Anderson O, Hedetoft M et al. SuPAR correlates with mortality and clinical severity in patients with necrotizing soft-tissue infections: results from a prospective, observational cohort study. Sci Rep. 2018;2019:1-8.

18. Hansen MB, Rasmussen LS, Garred P, Bidstrup D, Madsen MB, Hyldegaard O. Pentraxin-3 as a marker of disease severity and risk of death in patients with necrotizing soft tissue infections: a nationwide, prospective, observational study. Crit Care. 2016;20:40

19. Hansen MB, Rasmussen LS, Pilely K, Hellemann D, Hein E, Madsen MB, et al. The lectin complement pathway in patients with necrotizing soft tissue infection. J Innate Immun. 2016;8:507-16.

20. Hansen MB, Rasmussen LS, Svensson M, Chakrakodi B, Bruun T, Madsen $M B$, et al. Association between cytokine response, the LRINEC score and outcome in patients with necrotising soft tissue infection: a multicentre, prospective study. Sci Rep. 2017. https://doi.org/10.1038/srep42179.

21. Zhao T, Su Z, Li Y, Zhang X, You Q. Chitinase-3 like-protein-1 function and its role in diseases. Signal Transduct Target Ther. 2020;5:1-20. https://doi. org/10.1038/s41392-020-00303-7.

22. Hattori N, Oda S, Sadahiro T, Nakamura M, Abe R, Shinozaki K, et al. YKL-40 identified by proteomic analysis as a biomarker of sepsis. Shock. 2009;32:393-400

23. Kornblit B, Hellemann D, Munthe-Fog L, Bonde J, Strøm JJ, Madsen $\mathrm{HO}$, et al. Plasma YKL-40 and CHI3L1 in systemic inflammation and sepsis - experience from two prospective cohorts. Immunobiology. 2013;218:1227-34. https://doi.org/10.1016/j.imbio.2013.04.010.

24. Liu JP, Wang XW, Qie LP. Disease indicators for sepsis and analysis of sepsis treatment in children using the continuous blood purification technique. Genet Mol Res. 2015;14:5685-93.

25. Hansen MB, Simonsen U, Garred P, Hyldegaard O. Biomarkers of necrotising soft tissue infections: aspects of the innate immune response and effects of hyperbaric oxygenation-the protocol of the prospective cohort BIONEC study. BMJ Open. 2015;5: e006995.

26. Elm E, Altman D, Egger M, Stuart J, Gøtzsche P, Vandenbroucke J. The Strengthening the Reporting of Observational Studies in Epidemiology (STROBE) statement: guidelines for reporting observational studies. Lancet. 2007;370:1453-7.

27. Madsen MB, Skrede S, Bruun T, Arnell P, Rosen A, Nekludov M, et al. Necrotizing soft tissue infections-a multicentre, prospective observational study (INFECT): protocol and statistical analysis plan. Acta Anaesthesiol Scand. 2018;62:272-9.

28. Bojesen SE, Johansen JS, Nordestgaard BG. Plasma YKL-40 levels in healthy subjects from the general population. Clin Chim Acta. 2011;412:709-12.

29. Fluss R, Faraggi D, Reiser B. Estimation of the Youden Index and its associated cutoff point. Biom J. 2005;47:458-72.

30. Bernal NP, Latenser BA, Born JM, Liao J. Trends in 393 necrotizing acute soft tissue infection patients 2000-2008. Burns. 2012;38:252-60.

31. Gunter OL, Guillamondegui OD, May AK, Diaz JJ. Outcome of necrotizing skin and soft tissue infections. Surg Infect (Larchmt). 2008;9:443-50.

32. Jabbour G, El-Menyar A, Peralta R, Shaikh N, Abdelrahman H, Mudali IN, et al. Pattern and predictors of mortality in necrotizing fasciitis patients in a single tertiary hospital. World J Emerg Surg. 2016;11:1-10. https://doi. org/10.1186/s13017-016-0097-y.

33. Endorf FW, Supple KG, Gamelli RL. The evolving characteristics and care of necrotizing soft-tissue infections. Burns. 2005;31:269-73.

34. Hua C, Sbidian E, Hemery F, Decousser JW, Bosc R, Amathieu R, et al. Prognostic factors in necrotizing soft-tissue infections (NSTI): a cohort study. J Am Acad Dermatol. 2015;73:1006-12.e8.

35. Kao LS, Lew DF, Arab SN, Todd SR, Awad SS, Carrick MM, et al. Local variations in the epidemiology, microbiology, and outcome of necrotizing soft-tissue infections: a multicenter study. Am J Surg. 2011;202:139-45.

36. Elliott D, Kufera JMR. Necrotizing soft tissue infections : risk factors for mortality and strategies for management. Ann Surg. 2006;224:672-83.

37. Anaya DA, McMahon K, Nathens AB, Sullivan SR, Foy H, Bulger E. Predictors of mortality and limb loss in necrotizing soft tissue infections. Arch Surg. 2005;140:151-7; discussion 158

38. Kha P, Colot J, Gervolino S, Guerrier G. Necrotizing soft-tissue infections in New Caledonia: epidemiology, clinical presentation, microbiology, and prognostic factors. Asian J Surg. 2017;40:290-4.

39. Harrell FEJ, Lee KL, Mark DB. Multivariable prognostic models: issues in developing models, evaluating assumptions and adequacy, and measuring and reducing errors. Stat Med. 1996;15:361-87.

40. Peduzzi P, Concato J, Kemper E, Holford TR, Feinstein AR. A simulation study of the number of events per variable in logistic regression analysis. J Clin Epidemiol. 1996:49:1373-9.

41. Leligdowicz A, Conroy AL, Hawkes M, Zhong K, Lebovic G, Matthay MA, et al. Validation of two multiplex platforms to quantify circulating markers 
of inflammation and endothelial injury in severe infection. PLOS ONE. 2017;12:1-14

42. Kronborg G, Østergaard C, Weis N, Nielsen H, Obel N, Pedersen SS, et al. Serum level of YKL-40 is elevated in patients with Streptococcus pneumoniae bacteremia and is associated with the outcome of the disease. Scand I Infect Dis. 2002;34:323-6.

43. Spoorenberg SMC, Vestjens SMT, Rijkers GT, Meek B, van Moorsel CHM, Grutters JC, et al. YKL-40, CCL18 and SP-D predict mortality in patients hospitalized with community-acquired pneumonia. Respirology. 2017;22:542-50

44. Maddens B, Ghesquière B, Vanholder R, Demon D, Vanmassenhove J, Gevaert K, et al. Chitinase-like proteins are candidate biomarkers for sepsis-induced acute kidney injury. Mol Cell Proteomics. 2012;11:1-13.

45. Outinen TK, Mantula P, Jaatinen P, Hämäläinen M, Moilanen E, Vaheri A, et al. Glycoprotein YKL-40 is elevated and predicts disease severity in puumala hantavirus infection. Viruses. 2019;11:1-11.

46. Kjaergaard AD, Helby J, Johansen JS, Nordestgaard BG, Bojesen SE. Elevated plasma YKL-40 and risk of infectious disease: a prospective study of 94665 individuals from the general population. Clin Microbiol Infect. 2020:26:1411.e1-1411.e9. https://doi.org/10.1016/..cmi.2020.01.010

47. Bian B, Li L, Yang J, Liu Y, Xie G, Zheng Y, et al. Prognostic value of YKL-40 in solid tumors: a meta-analysis of 41 cohort studies. Cancer Cell Int. 2019;19:259.
48. Kzhyshkowska J, Mamidi S, Gratchev A, Kremmer E, Schmuttermaier C, Krusell L, et al. Novel stabilin-1 interacting chitinase-like protein (SI-CLP) is up-regulated in alternatively activated macrophages and secreted via Iysosomal pathway. Blood. 2006;107:3221-8.

49. Nielsen AR, Plomgaard P, Krabbe KS, Johansen JS, Pedersen BK. IL-6, but not TNF-a, increases plasma YKL-40 in human subjects. Cytokine. 2011;55:152-5. https://doi.org/10.1016/j.cyto.2011.03.014.

50. Hübner K, Karwelat D, Pietsch E, Beinborn I, Winterberg S, Bedenbender $\mathrm{K}$, et al. NF-KB-mediated inhibition of microRNA-149-5p regulates Chitinase-3-like 1 expression in human airway epithelial cells. Cell Signal. 2019;2020(67): 109498. https://doi.org/10.1016/j.cellsig.2019.109498.

51. Karwelat D, Schmeck B, Ringel M, Benedikter BJ, Hübner K, Beinborn I, et al. Influenza virus-mediated suppression of bronchial Chitinase-3-like 1 secretion promotes secondary pneumococcal infection. FASEB J. 2020;34:16432-48.

\section{Publisher's Note}

Springer Nature remains neutral with regard to jurisdictional claims in published maps and institutional affiliations.
Ready to submit your research? Choose BMC and benefit from:

- fast, convenient online submission

- thorough peer review by experienced researchers in your field

- rapid publication on acceptance

- support for research data, including large and complex data types

- gold Open Access which fosters wider collaboration and increased citations

- maximum visibility for your research: over 100M website views per year

At BMC, research is always in progress.

Learn more biomedcentral.com/submissions 